\title{
Development of a Platform to Explore Network Intrusion Detection System (NIDS) for Cybersecurity
}

\author{
Chee Keong Chan, Alexander Weil Tine Yeoh \\ School of Electrical and Electronic Engineering, Nanyang Technological University, Singapore \\ Email: eckchan@ntu.edu.sg
}

How to cite this paper: Chan, C.K. and Yeoh, A.W.T. (2018) Development of a Platform to Explore Network Intrusion Detection System (NIDS) for Cybersecurity. Journal of Computer and Communications, 6, 1-11.

https://doi.org/10.4236/jcc.2018.61001

Received: September 18, 2017 Accepted: December 26, 2017 Published: December 29, 2017

\begin{abstract}
Cybersecurity is increasing its significance in recent years due to the overwhelming use of devices which require the use of internet. This raises the importance of having cybersecurity training for the upcoming generations as hackers continue to upgrade their methodologies and techniques to obtain important information such as personal identification, credit card numbers etcetera. This paper describes the development of a platform for students to learn how to setup and use a Network Intrusion Detection System in a virtual environment. In this environment, the administrator of a specific system can monitor and detect their network for any malicious activity. We will discuss in this paper the network configuration setup via virtualization technology followed by having a Network Intrusion Detection System installed in one of the virtual machines port mirrored to monitor the whole network. In the virtual network, a virtual machine will be assigned as an attacker to simulate cyber-attacks allowing the Network Intrusion Detection System to detect the Internet Protocol (IP) address from the source of malicious activity provider. In addition, students will have the opportunity to learn how to write basic rules for the Network Intrusion Detection System which are algorithms used to detect cyber malicious movements.
\end{abstract}

\section{Keywords}

Network Intrusion Detection System, Cybersecurity

\section{Introduction}

Cyber security plays a vital role in protecting hardware, software and information which are important in our current century. Malicious actions such as 
hacking, stealing of information, damaging cyber components and disruption of services will have vast impact on our current society, which is heavily reliant on computer systems and the internet. Any such form of malicious actions will also have major repercussions on the world economy, which is very much interlinked through the internet. Hence, cybersecurity has a vital role to play to ensure the security and advancement of the global network.

Intrusion Detection System (IDS) is one of the counter measures against malicious activities in cyber security. The IDS is an equipment or software which checks a system or network for any harmful intention or violation of policies. The administrator of the system or network will be notified by the IDS in times of any suspected malicious activity found.

Intrusion Detection Systems are generally grouped into two main categories, the Host-based Intrusion Detection System (HIDS) and the Network Intrusion Detection System (NIDS). A HIDS monitors the operating system files such as the antivirus software whereby a NIDS monitors the incoming network traffic for any malicious activity [1].

The detection approaches are usually by signature-based detection and anomaly-based detection. Signature-based detection is a methodology where the software can identify intentions with harmful patterns such as malware. Anomaly-based detection monitors the offset of traffic from its ideal route, where it often depends on machine learning.

\section{Hyper-V Virtual Network Setup}

To start off students will learn to create a network for the Network Intrusion Detection System (NIDS) to monitor a specific network. In this case, Hyper-V which is a virtualization platform introduced by Microsoft [2] is utilized to create the virtual network. Having a virtual network over a physical network provides numerous advantages, such as cost reduction and less time needed to modify components on a computer. Hence a virtual network is a great compliment for learning NIDS. Next, the NIDS used in this environment is Snort, which is an open source IDS that can accomplish real time analysis and sniffing of network packets [3]. It will be installed into one of the virtual machines.

With the aid from Hyper- $\mathrm{V}$, four virtual machines will be created and they are termed as Server 1 to 4 respectively. These servers are connected internally via a virtual network interface controller (NIC). It simulates a network of four computers, eachconnected to a router with Ethernet cables. The four virtual machines will have their individual local static IP address setup for detection and verification. Server 1 to 4 will be assigned to the IP addresses of "192.168.2.1" to "192.168.2.4" respectively as shown in Figure 1.

With the virtual network setup, students will then have to identify if the servers are virtually internally connected by pinging each other. Ping is part of internet programming which allows the user to authenticate the existence of a IP address. Ping operates by sending an Internet Control Message Protocol (ICMP) Echo Request to a network interface and waits for its reply in which it is also 
Server 1

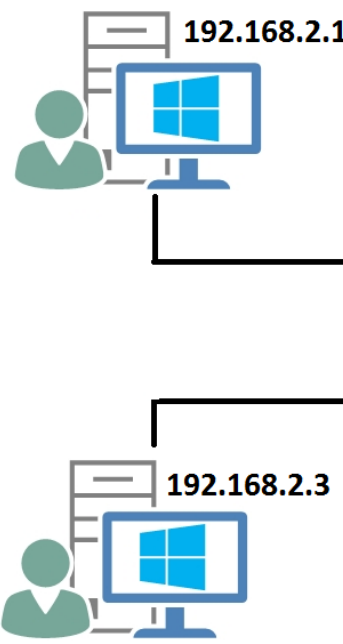

Server 3
Server 2

192.168.2.2
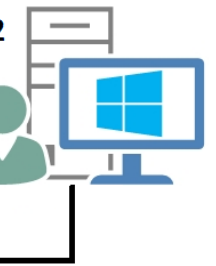

Figure 1. Virtual Network Diagram.

used to troubleshoot, test connectivity and identify response time between two IP addresses [4]. An example of Server 4 (192.168.2.4) issue a ping request towards Server 2 (192.168.2.2) with succession is shown in Figure 2 by command prompt where students will verify the virtual machine's IP address by the command:

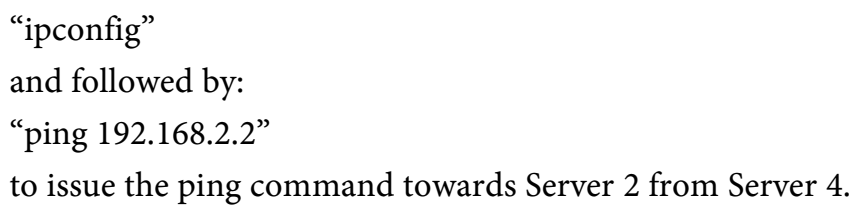

\section{Set up of SNORT and Writing Rule Basics}

After establishing the virtual network, the next step is to set up the Network Intrusion Detection System (NIDS) by using Snort in which for this instance Server 3 will be acting as the NIDS. Thus, Server 3 will no longer have its static IP address but rather it will be set to port mirror the other Servers to monitor the whole virtual network. Port Mirroring is a method whereby it duplicates all incoming and outgoing traffic to a configured destination [5] which is used to monitor the whole network. An overview of the updated virtual network is as shown in Figure 3.

Snort running in Windows similarly uses command prompt as its console to do certain tasks such as sniffing of network packet and showing of results. After a successful installation of Snort, the first and foremost important step is to do proper coding on the configuration file via Notepad++ such as setting up IP addresses to protect and pin pointing towards rule files which are files that consists of predefined algorithms written by open community and Snort's developers. These rules are created to identify various cyber-attacks and are being updated over the years to keep up with the ever-growing methodologies of cyber-attacks. 


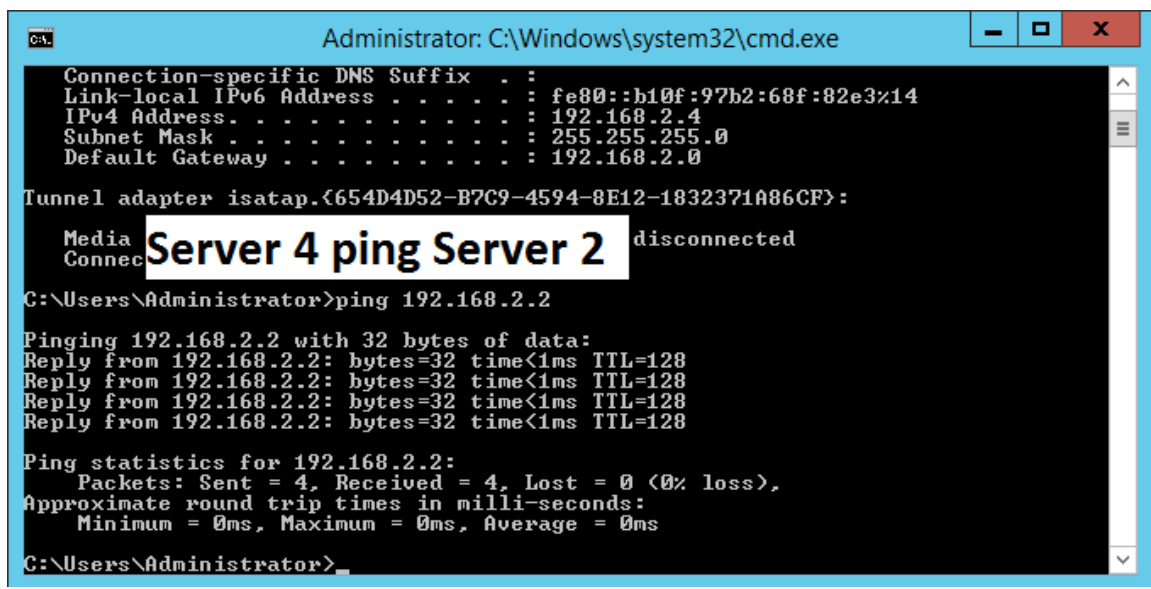

Figure 2. Server 4 ping to Server 2.

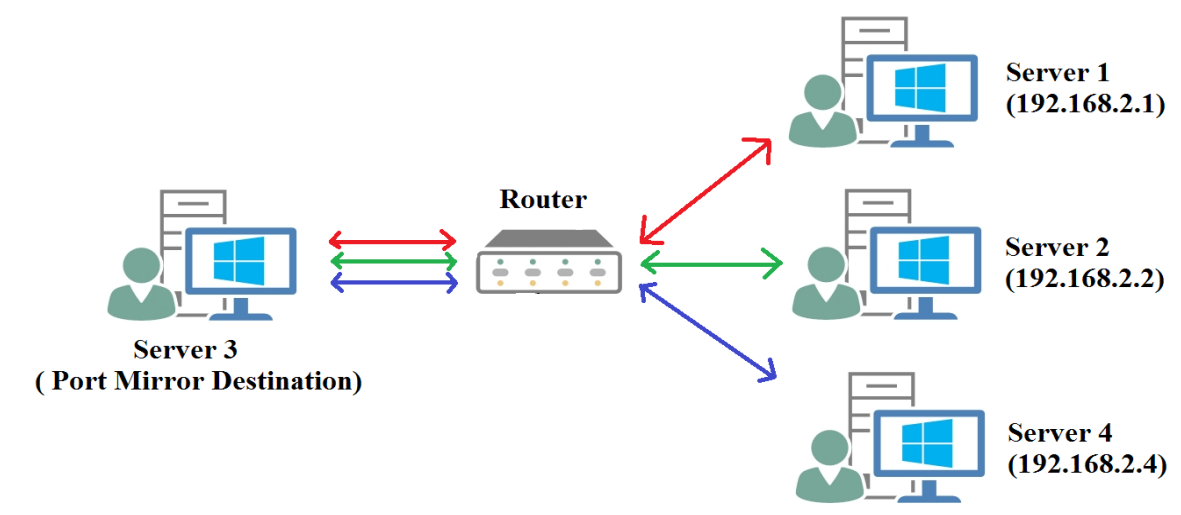

Figure 3. Virtual Network Diagram with NIDS.

With proper configuration, Snort should be tested first to rectify any compiling errors by using command prompt to first call the bin directory in Server 3 with "cd C:ISnort|bin" then executing the command:

"snort-i 1-c c:|Snortletclsnort.conf-T"

whereby "-i" displays which virtual interface card is used, “-c" calls the specific configuration file and "- $T$ " starts Snort in self-test mode. If no compiling errors are discovered, Snort is then ready to be used as a NIDS.

Students will then be required to write the first rule to verify if the NIDS is in working condition. An example of writing Snort's rule will be as follow:

"alert icmp \$EXTERNAL_NET any -> \$HOME_NET any (msg: "ICMP TEST"; sid: 100001;)"

- "alert"-The rule header whereby the "alert" command will generate an alert followed by logging the packet.

- "icmp"-The protocol defined for ping. Other available protocols such as tcp and udp are available as well.

- "\$EXTERNAL_NET"-The IP address assigned in the configuration file to detect external users which has been set as "\$HOME_NET" which means not equals to the protected network. This variable can be replaced by any specific IP address depending on the programmer. 
- "any"-The port variable for \$EXTERNAL_NET whereby for this case it will be any port as we are trying to verify the workability of the NIDS.

- "->-The arrow which indicates the traffic for the rule to verify. Whereby this case we are verifying for pings from variable \$EXTERNAL_NET towards \$HOME_NET.

- "\$HOME_NET"-The IP address assigned in the configuration file to protect.

- "any"-Similarly the port variable defined for the \$HOME_NET

- "msg"-A message to generate in the alert console when the NIDS has detected a similar pattern as defined by the specified rule. In this case, it will show a message of "ICMP TEST" when the rule is activated.

- "sid"-A simple ID to keep track of the number of rules which works similarly as serial number. The change of "sid" will not affect how to rule detects its pattern in the network traffic.

In a nutshell, the general form of a Snort rule is as follow:

(Rule Header) (Protocol) (IP Address 1) (Port Variable for IP Address 1) -> (IP Address 2) (Port Variable 2) (Methodologies defined to a specific pattern with message and SID)

The methodologies section may contain several algorithms to detect certain malicious such as having a threshold to track the number of incoming traffic received in a specific time set or detecting incoming traffic with different variants of Flags for instance "flags:S;" to detect SYN packet requests. There are numerous ways to define the rule and it is entirely up to the programmer to decide how the NIDS detects malicious traffic.

Once Snort is ready, the command:

"snort-i 1-c c:|Snortletclsnort.conf -A console"

is executed to activate Snort to monitor the network where "- $A$ " is used to set Snort into Alert Mode and "console" allows snort to run in console mode. Figure 4 shows an example of ICMP ping alert from Server 4 to Server 2 using Snort's console (IP address 192.168.2.4 $\rightarrow$ 192.168.2.2) when executing the same command in Figure 3 and defining \$HOME_NET as Server 2 (192.168.2.2) and \$EXTERNAL_NET as Server 4 (192.168.2.4).

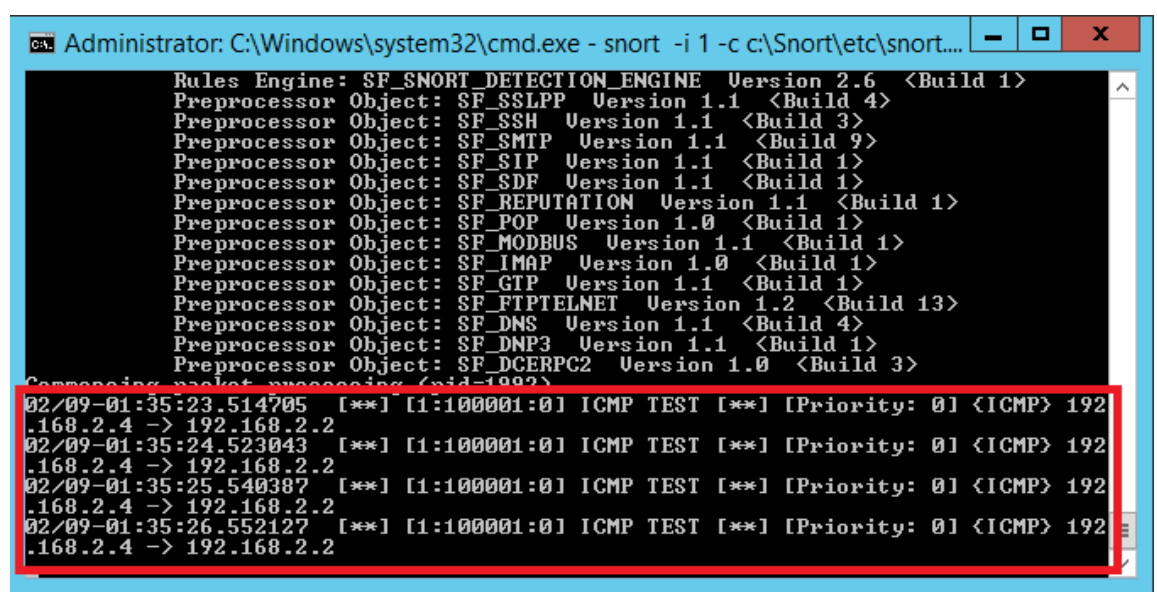

Figure 4. Snort rule ICMP alert test. 
The line "192.168.2.4 $\rightarrow$ 192.168.2.2" shows the network traffic flows from Server 4 towards Server2. In addition, the ICMP Ping alert will trigger as well when Server 1 pings Server 2 due to the rule's algorithm and how the IP address variables are defined. Once verified, the student has successful set up a Network Intrusion Detection System in a virtual network environment.

\section{Detecting Denial of Service Attacks}

Students will learn how to detect a Denial of Service (DOS) cyber-attack where attackers will flood the victim's server or network till it is too overwhelmed for the victim's resources and hence crashes its server. When the attacker uses multiple servers to attack a victim at once, it becomes a Distributed Denial of Service (DDOS).

WampServer will be installed on the protected Server which is Server 2 as initialised in Snort's configuration file. WampServer is a program which allows web hosting and development. Thus, Server 2 will be simulated as the victim of on an attack directly a web host. Using a web browser and calling Server 2 IP address will result a simple home page such as shown in Figure 5 below.

To simulate a DDOS attack Low Orbit Ion Cannon (LOIC) and the "old school" method Ping of Death (POD) will be used for learning purposes.

1) Low Orbit Ion Cannon (LOIC)

LOIC is a tool that performs DOS attack by sending large amounts of either Hypertext Transfer Protocol (HTTP), User Datagram Protocol (UDP) or Transmission Control Protocol (TCP) requests to a target destination [5]. LOIC was abused by a group of hackers who launched attack towards Paypal, Visa and Mastercard [6]. Hence the danger of this tool cannot be underestimated.

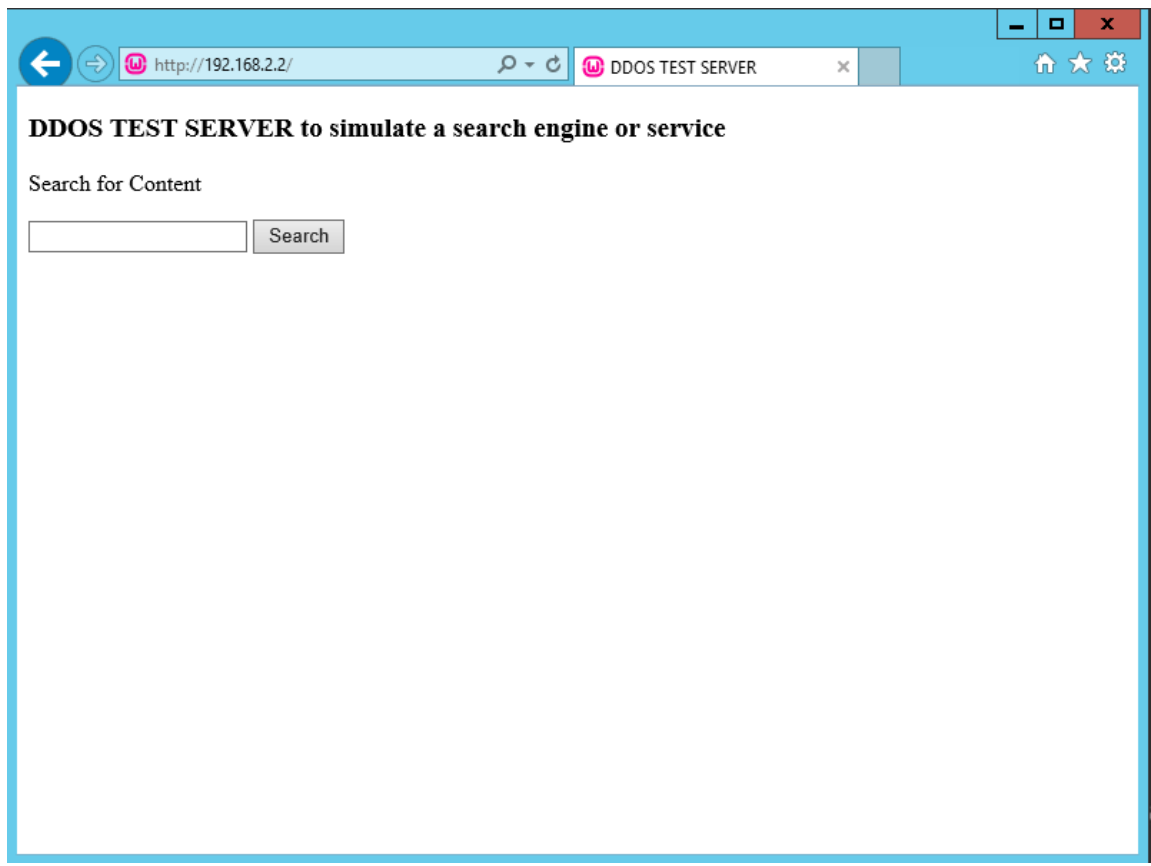

Figure 5. Sample local web host. 
The tool can simply be used by script kiddies to launch an attack by simply inputting the target's IP address and choosing the methodology of attack followed by clicking the "IMMA CHARGIN MAH LAZER" button to launch the attack. The effects of the attack will cause a heavy network traffic flow as shown on the victim's Ethernet network in Figure 6.

Snort rule used to detect TCP and HTTP attack method:

“alert tcp \$EXTERNAL_NET any -> \$HOME_NET any (msg: "LOIC DDOS TOOL ATTACK DETECTED”; threshold: type threshold, track by_src, count 100, seconds 5; sid:100002;)"

Threshold is a predefined algorithm that tracks from number of request sent from the host in counts of 100 within 5 seconds as LOIC works by sending several requests in a short amount of time.

Snort rule used to detect UDP attack method:

“alert udp \$EXTERNAL_NET any -> \$HOME_NET any (msg:”LOIC DDOS TOOL ATTACK DETECTED"; threshold: type threshold, track by_src, count 100 , seconds 5;sid:100003;)"

Like the TCP and HTTP attack method only a change in the Protocol from TCP to UDP.

During the attack, Snort's console should be able to detect the source of the attacker's IP address. In this simulation, Server 1 and 4 will be the attackers while Server 2 will be the victim. Figure 7 shows an example of the NIDS being able to capture LOIC's UDP attack from Server 1 and 4.

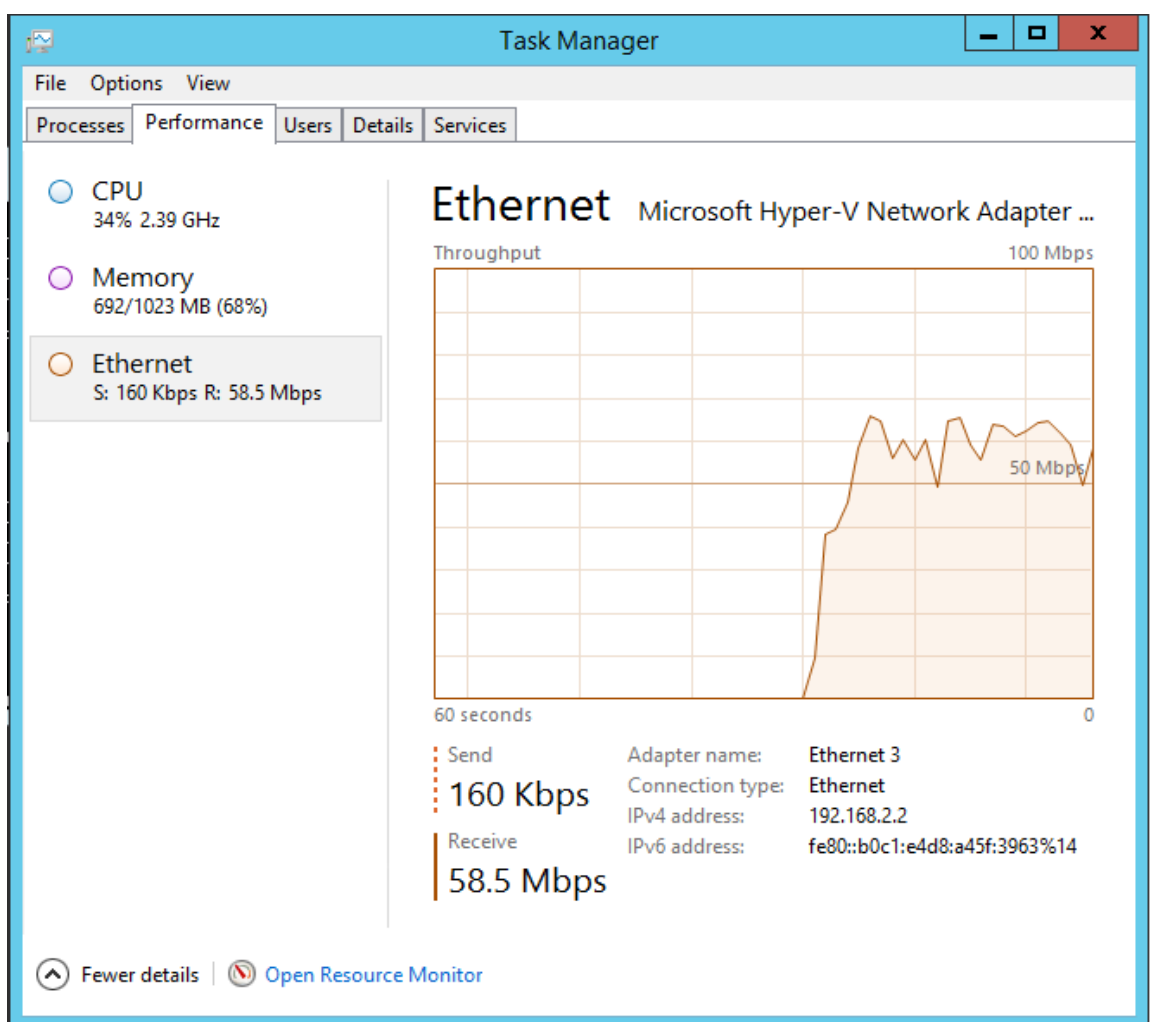

Figure 6. Effects of DOS Attack. 


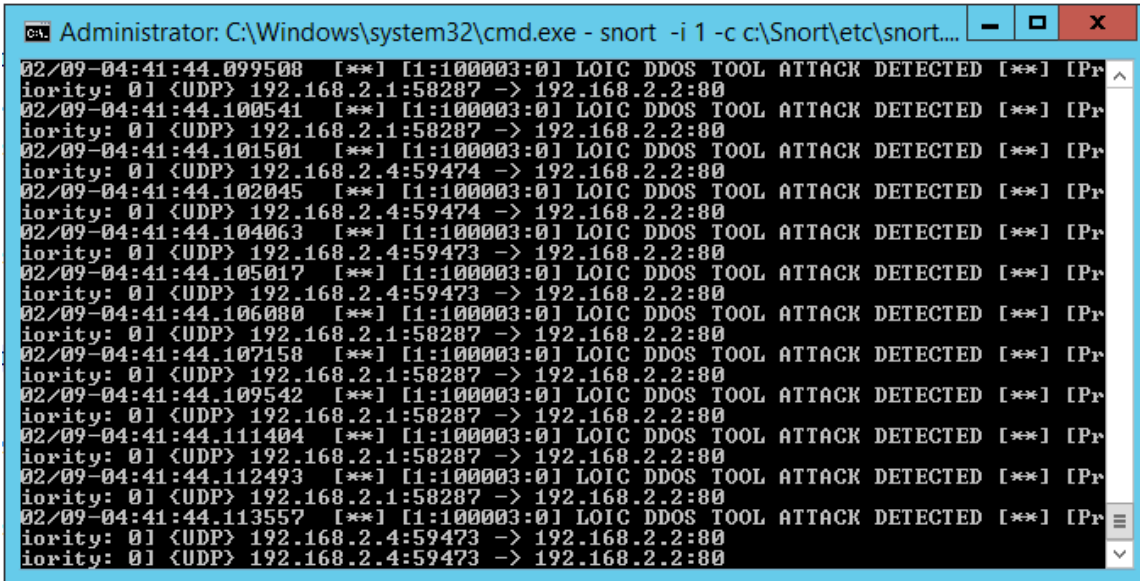

Figure 7. Snort detecting LOIC attacks.

2) Ping of Death (POD)

POD is a Denial of Service attack similar to sending an ICMP echo request (ping) except in large packet size and several occurrences at an instance.

The attack can be accomplished simply by using command prompt and using the command:

“ping 192.168.2.2 -t -1 65500”

"-t" instructs continuous echo requests until a stop command is issued while "-l" is used to define the size of the ping packet. Running this command in multiple instances will cause a similar result as shown previously in Figure 6 to the victim's server. Hence it is a form of Denial of Service attack.

Snort rule used to detect Ping of Death is as follow:

“alert icmp \$EXTERNAL_NET any -> \$HOME_NET any (msg: "PING OF DEATH DETECTED”; dsize:>1000; itype:8; icode:0; detection_filter:track by_src, count 30, seconds 1; sid:100004;)"

"dsize" is a keyword used to test the payload size, "itype" to check for an ICMP type value and "icode" to check for an ICMP code value. "detection_filter" works similarly to the threshold predefined algorithm in Snort.

In this simulation, Snort will be able to detect the attacks from both Server 1 and Server 4 while Server 2 will be the victim. Figure 8 shows an example of the results from Snort detecting Ping of Death attacks.

\section{Detecting Word and Word Content}

Cyber-attacks are usually from external sources. However there may be instances where employees unintentionally expose the company's network to unauthorised infiltration through careless use of the internal network. For instance, a seemingly harmless search for some information may open the door for some spyware to enter the company's network.

A Snort rule used to overcome this problem is as follow:

“alert tcp \$HOME_NET any -> \$EXTERNAL_NET any (msg:"WORD DETECTION"; content:"virus"; nocase; sid:100005;)" 


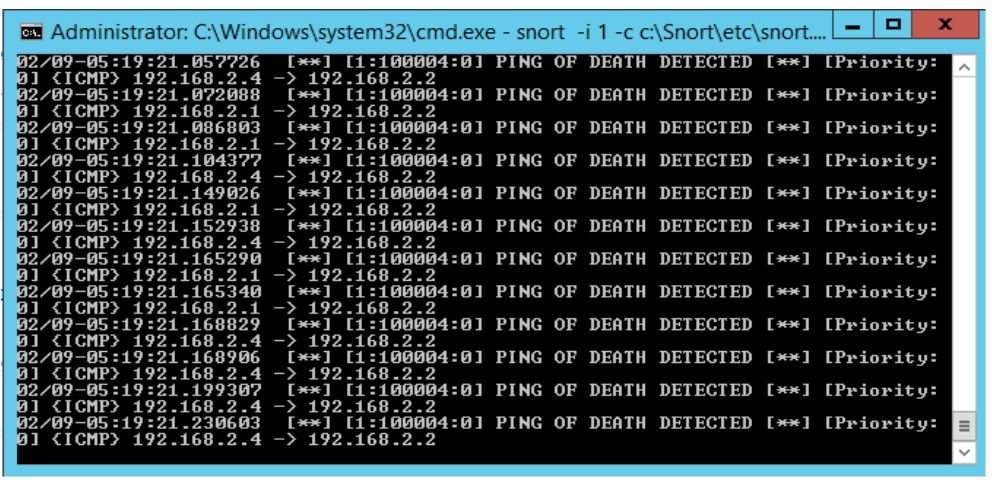

Figure 8. Snort detecting POD attacks.

Where "content" is a keyword the NIDS uses to check the data packet to monitor if any word "virus" is in the data packet while "nocase" basically supports the "content" keyword that the alphabetical case does not matter in detection.

In this simulation, Server 1 will be defined as "\$HOME_NET". Thus Server 2(Web Server) will be considered as "\$EXTERNAL_NET" reason being the configuration file, "\$EXTERNAL_NET" is defined as "\$HOME_NET". An example of Snort detecting Server 1 attempt to search for the keyword "virus" at Server 2 is shown in Figure 9.

\section{Detecting FTP Connectivity}

File Transfer Protocol (FTP) is a TCP network protocol service used to transfer files between a server and a client. Hackers may try to use this protocol to place malicious files into a server and cause harm. Hence it may be essential to identify who has access to the FTP Server.

In this simulation, Server 2 will be initialised as the FTP Server, while Server 1 will establish connection and place files into Server 2. From Server 1, by using command prompt and input the command:

"ftp 192.168.2.2"

Server 1 will try to establish a connection with the FTP Server. Once logged in, the next command:

"put (File name)"

Transfers the specified file from Server 1 to the FTP Server's database.

To detect FTP connectivity, the Snort rule used:

"alert tcp \$EXTERNAL_NET any -> \$HOME_NET 21 (msg: "INCOMING FTP DETECTED”; sid:100006;)"

The port number for " $\$$ HOME_NET" is specified as port 21 as the client by default usually creates a TCP connection using port number 21 . Hence, the NIDS will send an alert whenever there is activity from "\$EXTERNAL_NET" to “\$HOME_NET" in port 21 of the “\$HOME_NET" variable. Administrators have the choice to change or add more port numbers to the rule to enhance the security of the system. However, for this simulation case students will monitor port 21 only. Figure 10 shows the result of Snort detecting FTP connectivity between Server 1 and Server 2. 


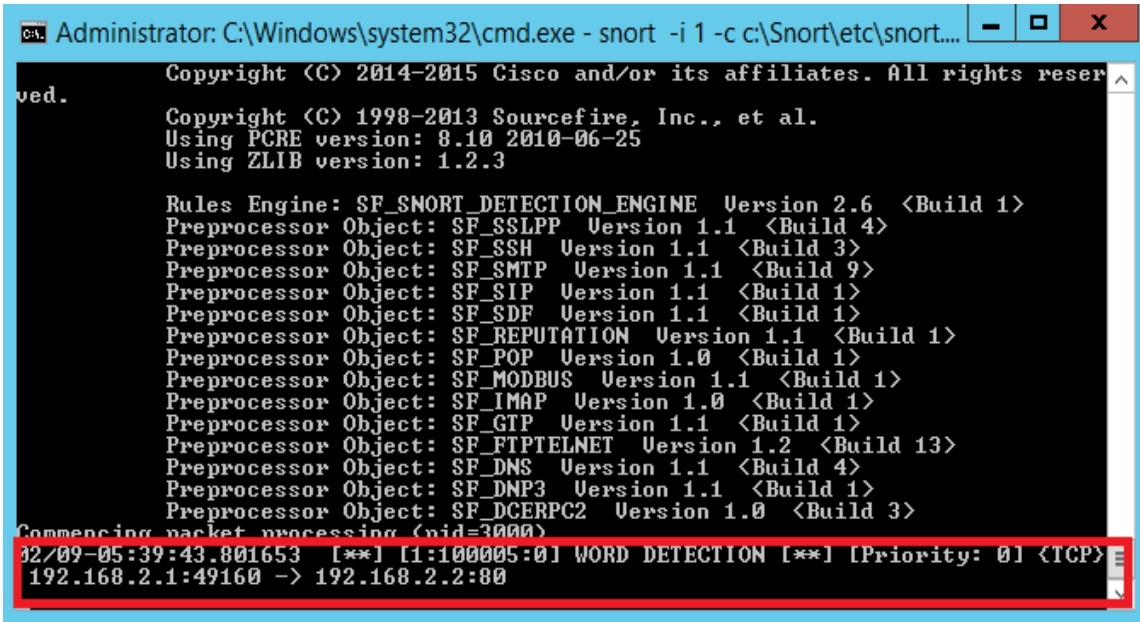

Figure 9. Snort detecting POD attacks.

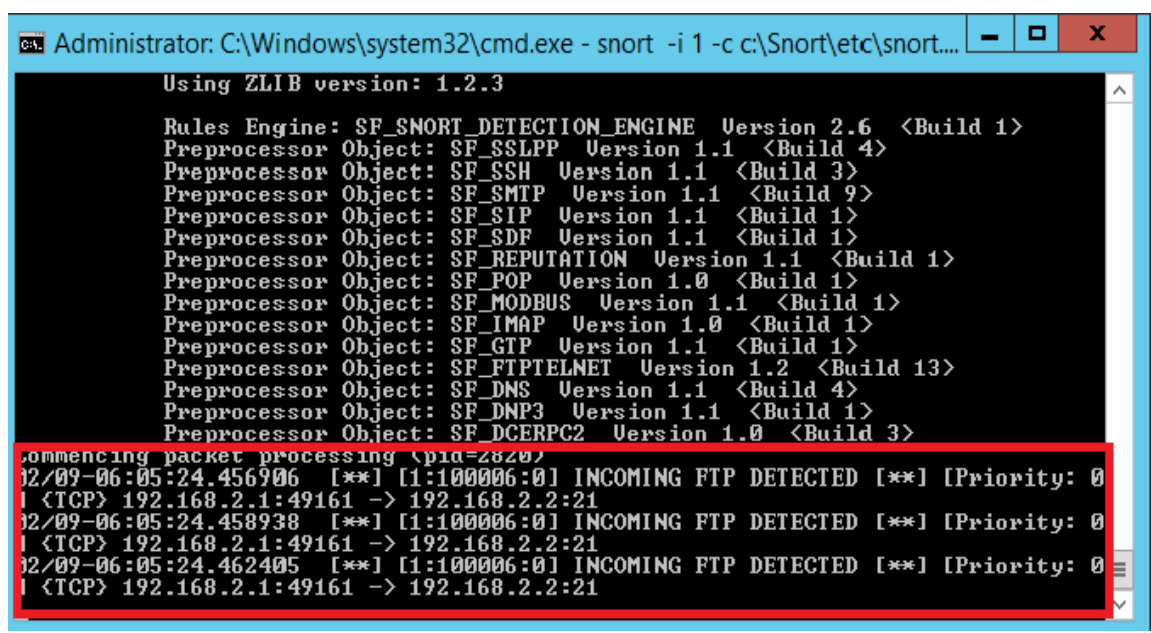

Figure 10. Snort detecting FTP connectivity.

\section{Conclusions}

With the setup of the Network Intrusion Detection System and examples of cyber-attacks, students will be able to gain more knowledge on virtualisation technology set ups and writing their own Snort rules base on the nature of the cyber-attacks. Students may now test their own NIDS over virtual labs, reaping a big reduction in cost and time and overcoming issues of purchasing and configuring hardware to conduct tests.

In addition, cyber attackers are always upgrading themselves and finding new methods to hack into systems. Hence it is important to keep ourselves updated with the current era to prevent our system from being jeopardised. With the basic knowledge gained, students now have a much easier time to explore further into writing more complicated rules and algorithms which could further pin point different forms of cyber-attacks. This would greatly enhance our capability to keep abreast of developments in the cyber-world, as well as reduce instances of false alerts during an intrusion detection. 


\section{References}

[1] Rouse, M. and Jaeger, R. (2008) HIDS/NIDS (Host Intrusion Detection Systems and Network Intrusion Detection Systems. Network Security.

[2] Zhelezko, A. (2014) What Is Hyper-V technology? Hyper-V Blog.

[3] Rouse, M. (2005) Snort. Network Security.

[4] Rouse, M. (2009) Ping. Internet Acronyms and Lingo.

[5] PRas, A., Sperotto, A., Moura, G.C.M., Drago, I., Barbosa, R., Sadre, R., Schmidt, R. and Hofstede, R. (2010) Attacks by "Anonymous" WikiLeaks Proponents Not Anonymous. Design and Analysis of Communication Systems Group (DACS) University of Twente, Enschede.

[6] Montoro, R. (2011) LOIC DDOS Analysis and Detection. SpiderLabs Blog. 